\title{
UBICACIÓN DE SITIOS APTOS PARA LA DISPOSICIÓN DE DESECHOS SÓLIDOS AL OESTE DEL VALLE CENTRAL, COSTA RICA
}

\author{
Elena Badilla, Wilfredo Rojas* \& Ingrid Vargas \\ Escuela Centroamericana de Geología, Universidad de Costa Rica, \\ Apdo. 214-2060, San Pedro \\ *Autor para contacto:wrojas@geologia.ucr.ac.cr
}

(Recibido: 30/04/07; aceptado 15/11/07)

\begin{abstract}
Suitable areas for the development of large scale landfill sites are defined, in order to help municipalities in coping with waste management problematic. The analysis is focused in an area located between Grande and Grande de Tárcoles rivers and Jesús María river, due to geological, economical and geographical reasons. Basic outlines are established to rule the suitability determination, considering distance to protected areas, rivers, access routes and towns, as well as topography, geology, hydrogeology, real estate conditions and land use regulations, among others. A preliminary site selection is carried out using GIS, combining binary factor maps with overlay functions. Three suitable sites larger than 50 ha are proposed, from which Vuelta Coyoles site, in Concepción, Atenas, shows better physical conditions and economical feasibility.
\end{abstract}

Keywords: Waste disposal, waste management, landfill site, Costa Rica, Great Metropolitan Area, GIS

\begin{abstract}
RESUMEN: Se definen sitios aptos para el desarrollo de grandes rellenos sanitarios regionales, con el fin de contribuir con las municipalidades en la búsqueda de una solución definitiva a la problemática del manejo de la basura. Por razones geológicas, económicas y de ubicación, el análisis se realiza en el área comprendida entre los ríos Grande - Grande de Tárcoles y Jesús María. Se establecen lineamientos básicos para determinar la aptitud de sitios para la disposición de desechos, considerando la distancia a áreas protegidas, ríos, rutas de acceso y poblados, así como la topografía, geología, hidrogeología, tenencia de la tierra y regulaciones territoriales, entre otros. Se lleva a cabo una selección preliminar de sitios con la ayuda de SIG, combinando mapas parámetro binarios mediante funciones de sobreposición. Se proponen tres sitios aptos con un área superior a 50 ha, de los cuales, el que presenta mejores condiciones físicas y de factibilidad económica es el sitio Vuelta Coyoles, en Concepción de Atenas.

Palabras clave: Relleno sanitario, manejo desechos sólidos, vertedero, Costa Rica, Gran Área Metropolitana, SIG
\end{abstract}

BADILLA, E., ROJAS, W. \& VARGAS, I., 2008: Ubicación de sitios aptos para la disposición de desechos sólidos al oeste del Valle Central, Costa Rica.- Rev. Geol. Amér. Central, 38: 7-19. 


\section{INTRODUCCIÓN}

Durante las últimas décadas, Costa Rica ha experimentado un marcado crecimiento en la cantidad de desechos sólidos que se generan anualmente, especialmente en la Gran Área Metropolitana (GAM). $\mathrm{Al}$ mismo tiempo, los actuales rellenos sanitarios o botaderos usados para verter estos desechos están alcanzando, o incluso superando, su vida útil.

Por mandato de la Sala Constitucional, el manejo y disposición final de los desechos es un asunto municipal. Sin embargo, los municipios y el Instituto de Fomento y Asesoría Municipal (IFAM), han demostrado y manifestado su imposibilidad para resolver el problema en el nivel que les corresponde.

No existe actualmente una labor municipal idónea de supervisión ni administración, en lo que respecta a esta temática. Además, la búsqueda de soluciones al problema de la basura ha sido insuficiente y la situación cada vez se torna más crítica, con el agravante de que la sociedad costarricense carece, en su mayoría, de hábitos positivos que faciliten el manejo de los desechos.

Hasta este momento, la legislación y reglamentación relacionada con los desechos sólidos en Costa Rica, determina que su disposición final mediante rellenos sanitarios, es técnicamente la alternativa más conveniente de disposición (La Gaceta No 206, 23 de octubre de 1998: Decreto 27378-S).

Dada la situación existente, este trabajo busca contribuir con las municipalidades en la búsqueda de una solución definitiva a esta problemática, especialmente en la GAM, a través de la definición de sitios aptos para el desarrollo de grandes rellenos sanitarios regionales (con capacidad mayor a 2000 ton/día), mediante la integración de estudios previos, nuevos conocimientos y la utilización de las recientes herramientas de los Sistemas de Información Geográfica (SIG).

Por las condiciones geológicas, hidrogeológicas, meteorológicas, geográficas y ambientales, las cuales se tratarán con detalle, así como por la accesibilidad a través de rutas nacionales y ferrocarril, el presente trabajo se enfocó en la búsqueda de posibles sitios al oeste del Valle Central.

\section{ANTECEDENTES}

En la década de los años 90, el Gobierno de la República, a través del Ministerio de Salud, el Ministerio de Asuntos Específicos y otras entidades como el Colegio de Médicos y Cirujanos, la Agencia de Cooperación Alemana (GTZ) y la Universidad de Costa Rica (UCR), efectuaron diagnósticos y prepararon documentos referentes a un plan nacional de manejo de desechos en el país. No fue hasta octubre de 1998 que se dictó, mediante el Decreto Ejecutivo No 27378-S (La Gaceta N No 206, 23 de octubre de 1998), el Reglamento Nacional de Manejo, Disposición y Operación de los Desechos Sólidos de Costa Rica.

Los estudios de la GTZ (1991), Knoblich \& Manz (1993), Steffen (1995), Astorga \& Avilés (1995), así como observaciones de otros investigadores (R. Flores, com. esc., 1995; L. Sánez, com. esc., 2005), coinciden en que los sitios aptos para la disposición de los desechos sólidos de la GAM deben localizarse en las zonas planas o semiplanas al oeste del Valle Central, preferiblemente en las plataformas ignimbríticas cuaternarias o las rocas de la Formación Aguacate, en los cantones de Mora, Orotina y Atenas, hacia el oeste de la confluencia entre el río Grande y el Virilla. De esta manera, no se impactarían los acuíferos Colima Inferior y Superior, Barva y Cebadilla.

Durante esta investigación se descartaron algunos sitios recomendados en estudios previos, debido a que carecen de acceso directo por vías nacionales o ferrocarril. Además, estos correspondían con localidades del cantón de Mora, donde el establecimiento de rellenos sanitarios para más de $300 \mathrm{t} / \mathrm{d}$, fue recientemente prohibido por el Concejo Municipal (La Gaceta N ${ }^{\circ} 50,10$ de marzo de 2006: Reglamento municipal sobre el manejo de residuos y rellenos sanitarios).

\section{DESCRIPCIÓN GENERAL DEL MÉTODO}

El proceso se inició con una revisión bibliográfica, cuyo objetivo fue establecer claramente los lineamientos básicos (requerimientos geológicos, 
hidrogeológicos, hidrológicos, geomorfológicos, arqueológicos, urbanísticos y ecológicos, establecidos por estudios previos y por normativas vigentes a nivel nacional) que se considerarían posteriormente en esta investigación, como guía para determinar la aptitud de sitios para la disposición de desechos sólidos al oeste del Valle Central (Cuadro 1). Esta revisión fue complementada con información sobre el avance de los planes reguladores que se encuentran en proceso, recabada especialmente en los cantones de Orotina y Atenas.

Se llevó a cabo además una recopilación de datos geológicos, hidrogeológicos y cartográficos digitales. Con estos se procedió a realizar una selección preliminar de sitios, aplicando técnicas de análisis espacial mediante la utilización del SIG Ilwis 3.3. Los datos base a partir de los cuales se trabajó fueron mapas de curvas de nivel cada $10 \mathrm{~m}$ del Proyecto Terra 1998, poblados, ríos y los trazos de la línea férrea y de la futura autopista San José - Caldera, digitalizados a partir de los mapas topográficos escala 1:50 000 del Instituto Geográfico Nacional (hojas Río Grande y Barranca), además del mapa de áreas protegidas del Atlas Costa Rica 2000 (Laboratorio de Sistemas de Información Geográfica EIF -ITCR, 2000), de los mapas geológicos de las hojas Río Grande (Arias \& Denyer, 1990) y Barranca (Denyer et al., 2003a), así como la localización de fallas neotectónicas obtenida de Montero (1999) y Denyer et al. (2003b).

Una vez obtenidas las áreas preliminares, se procedió a inspeccionarlas de manera general en el campo, considerando las características geológicas, ambientales, sociales, económicas y de uso del suelo. Esta inspección, combinada con la información técnica recabada, permitió corroborar la idoneidad de dichos sitios y por ende, la validez del modelo aplicado. Finalmente, se procedió a efectuar ensayos básicos de las propiedades geotécnicas y de infiltración de los suelos en los sitios seleccionados, con el fin de completar su descripción.

\section{LINEAMIENTOS GENERALES}

En el cuadro 1 se resumen los criterios de diversos autores, así como los incluidos en la normativa vigente a nivel nacional, sobre los lineamientos, parámetros, argumentos técnicos, físico-ambientales, sociales y económicos, involucrados en una adecuada selección y escogencia de sitios aptos para desarrollar rellenos sanitarios de índole regional.

\section{DEFINICIÓN DEL ÁREA DE ESTUDIO}

Como se dijo anteriormente, el presente trabajo se enfocó en la búsqueda de posibles sitios aptos para la ubicación de rellenos sanitarios al oeste del Valle Central, específicamente en aquellas áreas comprendidas entre los ríos Grande - Grande de Tárcoles y Jesús María, hojas topográficas Río Grande y Barranca, escala 1:50 000 del Instituto Geográfico Nacional (Fig. 1). Entre las razones por las cuales el estudio se centró en esta área están: la baja densidad de población actual, sus condiciones climatológicas, con estación seca bien marcada, poca exposición a vientos alisios por ser áreas de bajuras y resguardadas por colinas, la relativa poca presencia de parques nacionales, reservas y sitios arqueológicos, precios de terrenos razonables (comparados con los altos precios actuales de las propiedades hacia el interior del Valle Central), así como condiciones geológicas óptimas, como ya se indicó.

Otra razón determinante es la facilidad de acceso a esta área, tanto a través de la futura autopista San José - Caldera, como haciendo uso de la infraestructura ferroviaria existente, considerando además que al minimizar el kilometraje en transporte se reducen los costos (Astorga \& Avilés, 1995) y que el transporte por ferrocarril es de mucho menos costo económico ( $\phi 90 / \mathrm{t} / \mathrm{km}$ por carretera vs $\phi 35 / \mathrm{t} / \mathrm{km}$ por ferrocarril) y ambiental que por carretera. El INCOFER dispone actualmente de equipos e infraestructura para esta actividad (A. Dávila, Presidente Ejecutivo del Instituto Costarricense de Ferrocarriles (INCOFER) en ese momento, com. pers., 2005).

En caso de utilizarse el transporte por carretera, la futura autopista San José - Caldera representa la mejor opción, ya que se recomienda que el acceso a los rellenos sanitarios sea 
Cuadro 1

Lineamientos generales para la selección de sitios aptos para la disposición de desechos sólidos a nivel regional

Condiciones

Generales

Lineamientos

Los sitios no deben ubicarse sobre formaciones geológicas permeables, ni sobre rocas fracturadas, formaciones calcáreas con cavernas o corrientes subterráneas (GTZ, 1991).

En el área central de Costa Rica, las rocas que presentan condición favorable para este fin son las tobas de baja permeabilidad (Knoblich \& Manz, 1993).

Se deben evitar aquellas zonas con alto riesgo sísmico, peligrosas por la presencia de fallas tectónicas o por su susceptibilidad a deslizamientos (GTZ, 1991). En este punto cabe agregar que la presencia directa de fallas geológicas activas, representa un alto potencial de ruptura, lo cual puede afectar e impactar directamente el asiento de vertederos, romper los sellos inferiores y causar infiltración de lixiviados hacia el subsuelo o fracturamientos de la estructura del relleno, rompiéndose los sellos

1. Geología externos y liberándose gases y sustancias que contaminen el aire.

El sitio del vertedero debe estar a más de $60 \mathrm{~m}$ de las trazas de fallas geológicas activas (La Gaceta No 206, 23 octubre 1998: Decreto 27378-S).

Se aplicará en primera instancia la selección de la alternativa de diseño que evite pasar por el trazo de falla geológica activa y su respectiva zona de seguridad establecida según estudio geológico - neotectónico. (La Gaceta No 85, 4 mayo 2006: Decreto 32967-MINAE, Anexo 3).

El sitio no debe ubicarse sobre deslizamientos activos o que puedan reactivarse en el futuro (Van Westen, 1997; La Gaceta No 206, 23 octubre 1998: Decreto 27378-S), ni en áreas costeras susceptibles a inundaciones, planicies aluviales o áreas sujetas al efecto de los ríos, según los cambios estacionales (GTZ, 1991).

El relleno sanitario debe ubicarse a una distancia que garantice que las zonas de recarga de acuíferos y las fuentes subterráneas de abastecimiento de agua potable (pozos o manantiales), estén libres de contaminación (La Gaceta N$^{\circ}$ 206, 23 octubre 1998: Decreto 27378-S). Cabe indicar que la distancia preventiva mínima dependerá de las condiciones hidrogeológicas locales, ya que si bien es cierto algunas áreas de recarga en el Valle Central ya han sido identificadas, se presume que muchos ríos también pueden aportar agua a los acuíferos, por lo que garantizar que las fuentes de agua estén libres de contaminación, no es físicamente posible.

El suelo debe reunir características de impermeabilidad, remoción de contaminantes y profundidad del nivel de aguas subterráneas que garanticen la conservación de acuíferos de la zona (La Gaceta

\section{Aguas subterráneas} y superficiales No 206, 23 octubre 1998: Decreto 27378-S). Es importante agregar que la remoción dependerá del tipo y de la carga de contaminantes, ya que algunos suelos tienen una capacidad limitada para retener contaminantes y degradarlos, mientras que otros no la tienen del todo; en el país no existen estudios sobre estos aspectos.

Las tobas reúnen estas condiciones favorables (Knoblich \& Manz, 1993).

Si hay un acuífero, tiene que existir una barrera impermeable que impida el flujo contaminante de líquidos lixiviados (GTZ, 1991).

En lo que respecta a cuerpos de agua superficiales (riachuelos, ríos), debe acatarse lo estipulado en el Reglamento de Rellenos Sanitarios (La Gaceta No 206, 23 octubre 1998: Decreto 27378-S), el cual establece en el Artículo 7, inciso d, que la distancia a guardar, debe ser definida por la Dirección de Protección al Ambiente Humano del Ministerio de Salud.

En el desarrollo de los proyectos de rellenos sanitarios, debe mitigarse la degradación del paisaje, 3. Paisaje mediante la utilización de sitios que no estén expuestos visualmente en partes altas y mediante obras periféricas de reforestación (Astorga \& Avilés, 1995).

\section{Geomorfología y} topografía

Se recomienda su desarrollo en mesetas o montañas con áreas planas o de poca inclinación, lo que facilita el drenaje fácil y natural. Además, el fondo del sitio debe tener una pendiente mínima de $3 \%$ hacia las líneas de los tubos de drenaje y se deben evitar canteras y minas sin drenajes (GTZ, 1991). 


\begin{tabular}{|c|c|}
\hline $\begin{array}{l}\text { 5. Capacidad y vida } \\
\text { útil }\end{array}$ & $\begin{array}{l}\text { El área y capacidad del sitio seleccionado debe garantizar una vida útil superior a los diez años ( } L a \\
\left.\text { Gaceta } \mathrm{N}^{\circ} 206,23 \text { octubre 1998: Decreto } 27378-\mathrm{S}\right) \text {, preferiblemente de más de } 20 \text { años, conside- } \\
\text { rando un volumen de desechos de } 150 \text { t/d (GTZ,1991). }\end{array}$ \\
\hline \multirow[t]{2}{*}{$\begin{array}{l}\text { 6. Zonas protegidas, } \\
\text { arqueológicas o } \\
\text { pobladas }\end{array}$} & $\begin{array}{l}\text { El relleno sanitario no debe ubicarse dentro ni cerca de sitios de interés arqueológico, paleontológico } \\
\text { o ecológico. Debe localizarse a una distancia mínima de } 500 \text { m de áreas pobladas (GTZ, 1991). }\end{array}$ \\
\hline & $\begin{array}{l}\text { El coeficiente de permeabilidad (K) de los suelos en el sitio debe ser del orden de 10-7 m/s (Knoblich } \\
\text { \& Manz, 1993), mientras que sus espesores deben superar los } 3 \mathrm{~m}(\mathrm{GTZ}, 1991) .\end{array}$ \\
\hline \multirow[t]{2}{*}{ 7. Geotecnia } & $\begin{array}{l}\text { Se recomiendan, para los suelos en el sitio, valores para el límite líquido de } 35-45 \% \text {, para el límite } \\
\text { plástico de } 20-45 \% \text { y para el índice de plasticidad de } 10-25 \% \text {, característicos en suelos de baja plas- } \\
\text { ticidad, ó } 30-40 \% \text { en suelos mezclados arcilla/limo (Knoblich \& Manz, 1993) }\end{array}$ \\
\hline & Debe existir disponibilidad de material impermeabilizante (Astorga \& Avilés, 1995). \\
\hline
\end{tabular}

una carretera con dimensiones de autopista y soporte de carga, que garantice seguridad y un mínimo de congestionamiento durante todo el trayecto (GTZ, 1991).

\section{SELECCIÓN PRELIMINAR DE SITIOS}

Para la selección preliminar de sitios aptos para ubicación de rellenos sanitarios, se llevó a cabo una combinación de mapas parámetro binarios ( 2 únicos valores posibles: apto equivale a 1 / no apto equivale a 0 ), aplicando técnicas de análisis espacial, mediante la utilización del SIG Ilwis 3.3, el cual se aplicó a aquellas áreas comprendidas entre los ríos Grande - Grande de Tárcoles y Jesús María (Fig. 1).
Los mapas parámetro fueron creados a partir de los siguientes criterios:

Criterio áreas protegidas: se generó una zona de amortiguamiento (buffer) de $500 \mathrm{~m}$ (mismo criterio de GTZ (1991) para el caso de áreas pobladas) alrededor de todas las áreas protegidas comprendidas dentro del área de estudio. Con el fin de evitar posibles impactos ecológicos y arqueológicos, los sitios seleccionados no pueden encontrarse dentro de esta zona (distancia $>500$ $\mathrm{m}=$ apto, distancia $<500 \mathrm{~m}=$ no apto).

Criterio rutas de acceso: para garantizar la facilidad de acceso y la reducción de gastos de transporte, los sitios seleccionados deben localizarse a menos de $1 \mathrm{~km}$ de distancia de la futura autopista San José - Caldera y de la línea férrea (distancia < $1 \mathrm{~km}=$ apto, distancia $>1 \mathrm{~km}=$ no apto).

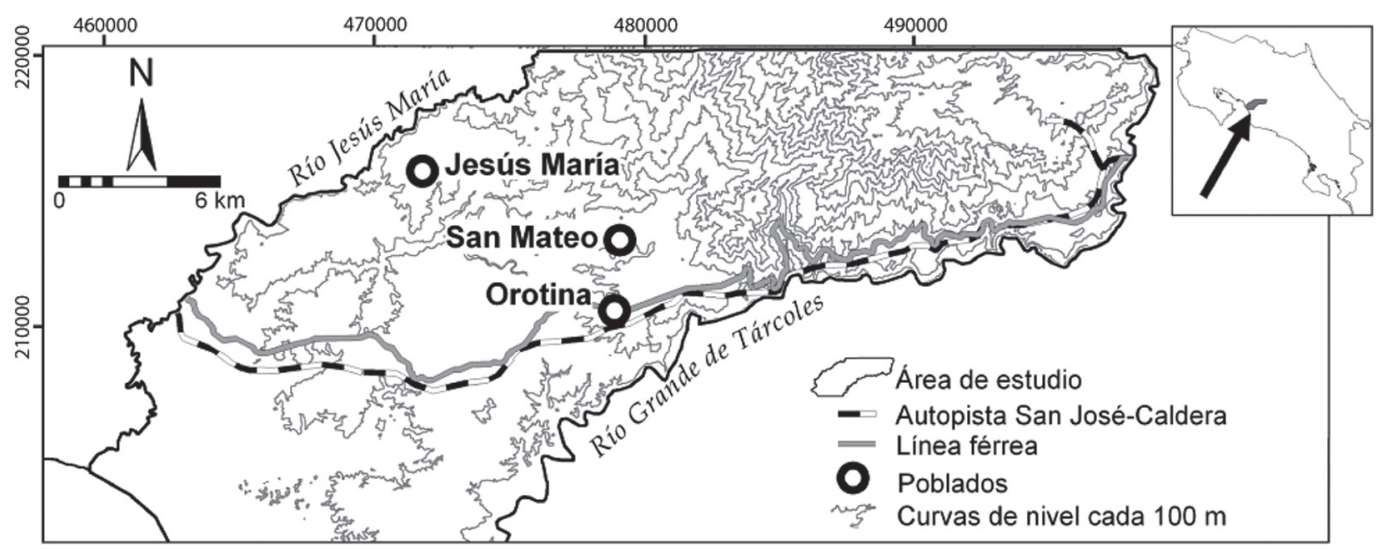

Fig. 1: Ubicación del área de estudio 
Criterio poblados: con el fin de minimizar el efecto del relleno en la población, los sitios seleccionados no pueden encontrarse a menos de 500 $\mathrm{m}$ de distancia de los principales centros poblados (distancia $>500 \mathrm{~m}=$ apto, distancia $<500 \mathrm{~m}=$ no apto). Este criterio corresponde con el planteado por GTZ (1991).

Criterio ríos: se generó una zona de amortiguamiento de $40 \mathrm{~m}$ a ambos lados de los ríos y principales quebradas del área de estudio (distancia tomada como referencia para este trabajo, ya que fue la recomendada por el Ministerio de Salud para el caso del actual relleno sanitario de la Carpio, con respecto al cauce del río Virilla, según se verificó en una gira a dicho proyecto). Los sitios seleccionados no pueden encontrarse dentro de esta zona, ya que de lo contrario se pondría en riesgo la calidad de los cuerpos de agua superficiales (distancia $>40 \mathrm{~m}=$ apto, distancia $<40 \mathrm{~m}=$ no apto).

Criterio topografía: a partir de las curvas de nivel se creó un modelo de elevación digital, del cual se derivaron las pendientes del terreno. Se estableció que los sitios seleccionados no pueden encontrarse en terrenos con pendientes superiores a los $10^{\circ}$ ya que, según se indicó anteriormente, se recomienda que se localicen en áreas planas o de poca inclinación (pendiente $<10^{\circ}=$ apto, pendiente $>10^{\circ}=$ no apto).

Criterio fallas: a pesar de que la normativa nacional establece que el sitio del vertedero debe estar a más de $60 \mathrm{~m}$ de las trazas de fallas geológicas activas (La Gaceta N N 206, 23 octubre 1998: Decreto 27378-S), en este trabajo se consideró esta zona de amortiguamiento como insuficiente para el área de estudio, ya que de acuerdo con Montero (1999), ésta se encuentra afectada por fallas neotectónicas de gran importancia como la Jesús María y el sistema de falla Tárcoles. Con base en esto, se estableció que los sitios seleccionados no pueden encontrarse a menos de $500 \mathrm{~m}$ de distancia de fallas neotectónicas (distancia $>$ $500 \mathrm{~m}=$ apto, distancia $<500 \mathrm{~m}=$ no apto) .

Criterio plan regulador: a pesar de no encontrase vigente aún, el futuro plan regulador del cantón de Orotina establecerá que las zonas ubicadas hasta $12 \mathrm{~km}$ al oeste del centro de Orotina serán destinadas a desarrollo industrial y residencial (Programa de Investigación en Desarrollo Urbano Sostenible (PRODUS-UCR), com. esc., 2006). Previendo esto, se ha considerado para este estudio que los sitios seleccionados no pueden encontrarse dentro del área afectada por esta regulación (en área regulada $=$ no apto, fuera de área regulada $=$ apto)

La combinación de estos mapas parámetro binarios mediante funciones de sobreposición (producto simple considerando apto $=1$ y no apto $=0$ ), dio como resultado un conjunto numeroso de zonas para las cuales todos los criterios cumplían la categoría de aptos (1337 sitios). De este primer grupo de resultados se descartaron todos aquellos sitios con un área menor a 50 ha, superficie necesaria para garantizar los 10 años de vida útil que exige el Reglamento de Rellenos Sanitarios (La Gaceta N ${ }^{\circ}$ 206, 23 octubre 1998: Decreto 27378-S). De este modo la lista de opciones se redujo a únicamente 3 sitios probables (Fig. 2).

Cabe destacar que la geología no fue incluida como criterio espacial de selección, ya que de acuerdo con Arias \& Denyer (1990) y Denyer et al. (2003a), el área de estudio se encuentra en su totalidad en rocas aptas para el desarrollo de proyectos de este tipo (Grupo Aguacate, Avalancha Ardiente, Formación Esparta y Formación Tivives). Se prefirió corroborar la idoneidad litológica de los sitios seleccionados directamente en el campo.

\section{CORROBORACIÓN DE APTITUD DE LOS SITIOS EN EL CAMPO}

Como se explicó anteriormente, una vez que se obtuvieron las áreas preliminares, se hizo una inspección general en el campo de sus características geológicas, hidrogeológicas, ambientales, sociales, económicas y de uso del suelo. Esto implicó la corroboración de los siguientes aspectos:

- Geología local

- Indicios de deslizamientos o de zonas inundables

- Disponibilidad de material impermeabilizante y de cobertura

- Consulta al Ministerio de Ambiente y Energía (MINAE) y al Museo Nacional sobre las 


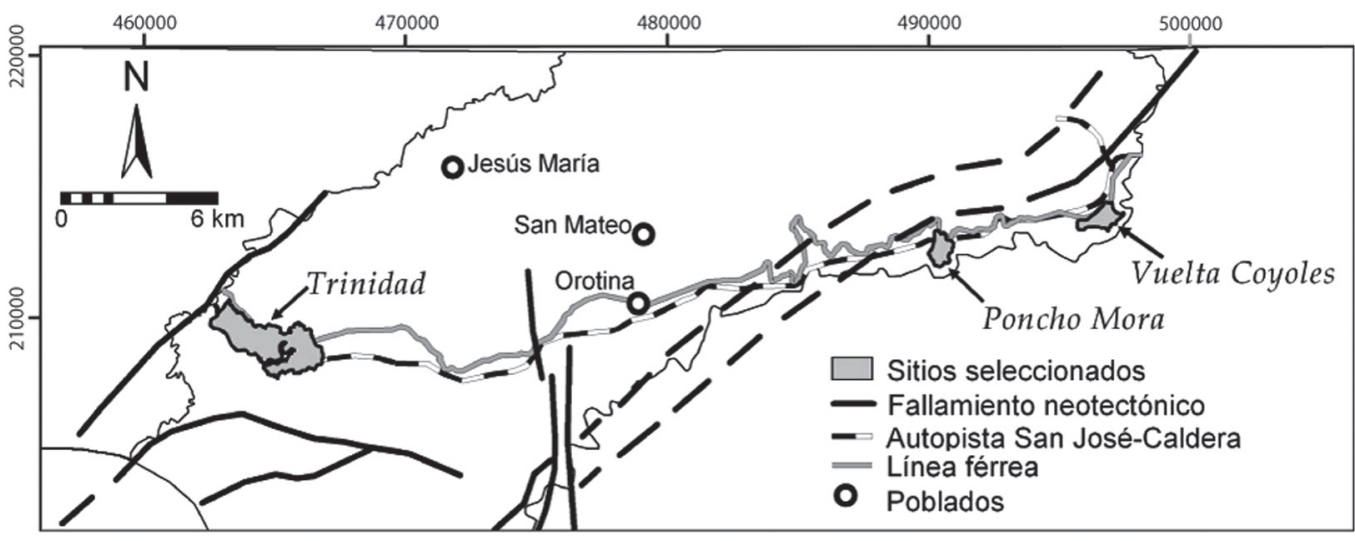

Fig. 2: Ubicación de los sitios seleccionados y del fallamiento neotectónico presente en el área (Montero, 1999 y Denyer et al., 2003b)

condiciones de los sitios en lo que respecta a áreas protegidas y de interés arqueológico.

- Consulta a los propietarios sobre su anuencia a vender dichas tierras y bajo qué condiciones de pago.

- Acceso simultáneo al trazo de autopista San José - Caldera y al ferrocarril.

- Localización en zonas de bajura, rodeados de colinas o montañas, y de bajo impacto visual y paisajístico.

- Existencia de manantiales que evidenciaran la presencia de aguas subterráneas someras, para lo que se inspeccionaron los sitios directamente en el campo y se consultaron personeros de la oficina de acueductos de los cantones de Orotina y Atenas, así como el registro de concesiones del Departamento de Aguas del MINAE
- Presencia y cercanía de pozos excavados y perforados en las zonas de interés, para lo que se consultó el Archivo Nacional de Pozos del SENARA.

- Predominio de vientos con dirección oeste, con velocidades que oscilen entre los 5 y los 40 $\mathrm{km} / \mathrm{h}$ (máximo en épocas ventosas de diciembre y enero), de manera que, por su ubicación, los sitios propuestos no afecten directamente ciudades o cabeceras de cantón.

Una vez corroborados estos aspectos en el campo, se determinó que lo tres sitios seleccionados preliminarmente cumplen con la totalidad de los requisitos propuestos, de manera que pueden considerarse como aptos, en principio, para la ubicación de rellenos sanitarios (Cuadro 2).

\section{Cuadro 2}

Características generales de los sitios seleccionados

\begin{tabular}{|c|c|c|c|c|c|c|c|}
\hline $\begin{array}{l}\text { Nombre del } \\
\text { sitio }\end{array}$ & $\begin{array}{c}\text { Área } \\
\text { (ha) }\end{array}$ & Vida útil * & $\begin{array}{c}\text { Distancia al } \\
\text { FEP ** }\end{array}$ & $\begin{array}{l}\text { Distancia a línea } \\
\text { férrea }(\mathrm{m})\end{array}$ & $\begin{array}{l}\text { Distancia a auto- } \\
\text { pista (m) }\end{array}$ & Relieve & $\begin{array}{l}\text { Precio } \\
\$ / \mathrm{m}^{2}\end{array}$ \\
\hline Vuelta Coyoles & 92 & 25 años & $40 \mathrm{~km}$ & 25 & 250 & Semiplano & $\begin{array}{c}2,0 \\
-10,0\end{array}$ \\
\hline Poncho Mora & 67 & 20 años & $47 \mathrm{~km}$ & 25 & 0 & Plano & $\begin{array}{c}3,0 \\
-7,70\end{array}$ \\
\hline Trinidad & 555 & 40 años & $81 \mathrm{~km}$ & 20 & 20 & Plano & $\begin{array}{c}2,0 \\
-3,0\end{array}$ \\
\hline
\end{tabular}

* Vida útil aproximada para $2000 \mathrm{t} / \mathrm{d}$

** Distancia aproximada a la Estación del Ferrocarril al Pacífico en San José 


\section{DESCRIPCIÓN GENERAL DE LOS SITIOS}

En general, los tres sitios propuestos en esta investigación como aptos para el desarrollo de rellenos sanitarios, se caracterizan desde el punto de vista geológico e hidrogeológico por litologías de baja permeabilidad y por presentar, en general, niveles freáticos localizados a más de $10 \mathrm{~m}$ profundidad; estos podrían corresponder con acuíferos colgados, sin embargo deben estudiarse con más detalle. El grado de meteorización de las litologías encontradas ha facilitado el desarrollo de suelos arcillosos, apropiados como sello y cobertura en estos rellenos. Además, en lo que respecta a características tectónicas, no se han encontrado fallas activas a menos de $100 \mathrm{~m}$ de ninguno de los tres sitios (Fig. 2 y Cuadro 2).

El Sitio Vuelta Coyoles (Figs. 2 y 3), conocido también como "El Cuarenta", se ubica en el cantón de Atenas, distrito Concepción, 4,5 km al sureste de la ciudad de Atenas, a una altitud entre 450 y 500 m s.n.m. y sobre ignimbritas de Avalancha Ardiente (Arias \& Denyer, 1990). Presenta un nivel freático a $11 \mathrm{~m}$ de profundidad (según pozo excavado en el sitio) y un espesor de suelo arcilloso observado en el sitio superior a los $3 \mathrm{~m}$ (Cuadro 3). Según datos del Archivo Nacional de Pozos del SENARA (Cuadro 4), los pozos registrados se encuentran a más de $1 \mathrm{~km}$ de distancia del sitio y en ellos se reportan niveles freáticos que oscilan entre 4 y $14 \mathrm{~m}$ de profundidad, con caudales de 0,5 y 3 1/s, cuando captan aguas almacenadas en tobas e ignimbritas; los caudales superan los 4 l/s en las lavas.

El Sitio Poncho Mora (Figs. 2 y 3), conocido también como "Planicie Los Mangos", se ubica en el cantón de Atenas, distrito Escobal, 6,5 km al suroeste de la ciudad de Atenas, a una altitud entre 370 y 390 m s.n.m. Presenta un nivel freático a más de $25 \mathrm{~m}$ de profundidad y el espesor promedio de suelo arcilloso es de $1 \mathrm{~m}$ (Cuadro 3). La geología en superficie es similar al Sitio Vuelta Coyoles (Arias \& Denyer, 1990). Según el Archivo Nacional de Pozos del SENARA (Cuadro 5), existen muy pocos pozos en sus alrededores, encontrándose los más cercanos a más de $2 \mathrm{~km}$ de distancia. Los niveles freáticos se ubican a más de $12 \mathrm{~m}$ de profundidad, excepto por el pozo RG-692 localizado a $3,2 \mathrm{~km}$ al noreste, en donde el nivel
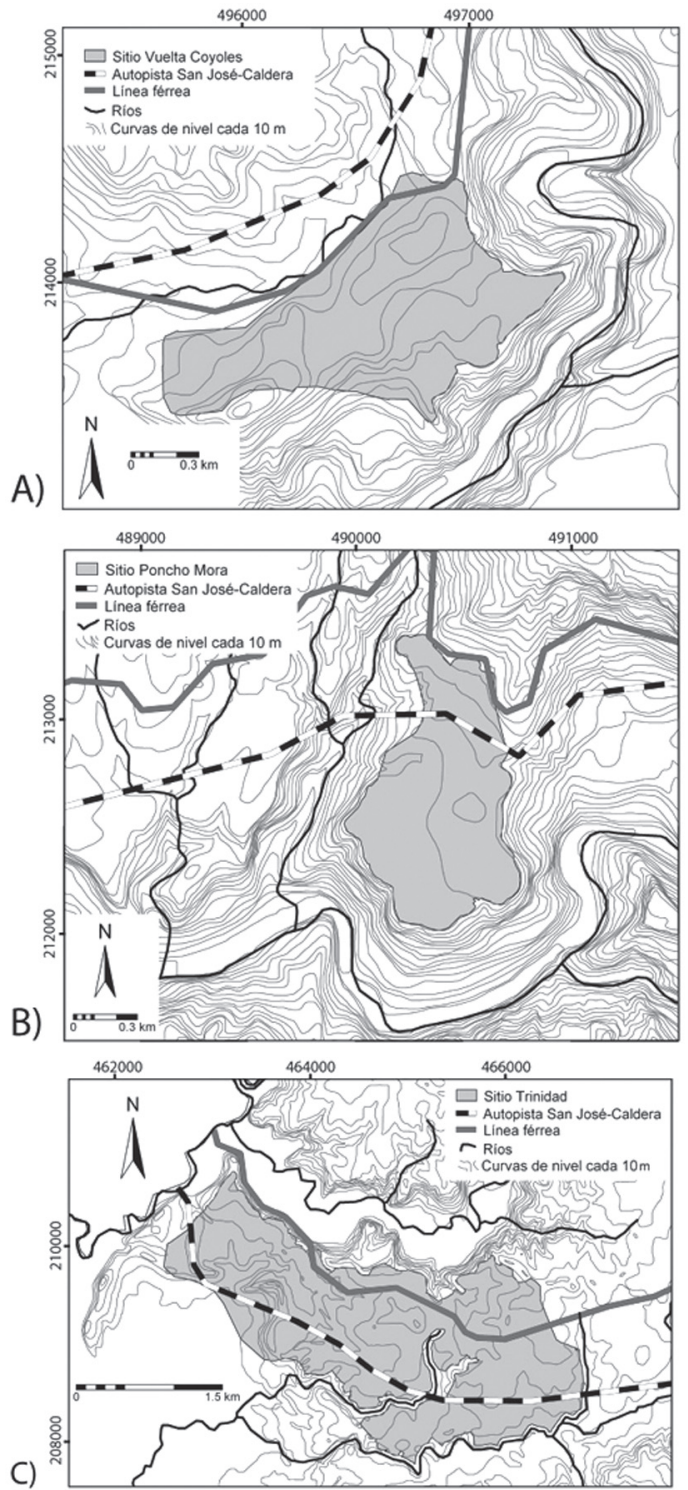

Fig. 3: Detalle de la topografía de los sitios seleccionados y su ubicación con respecto a rutas de acceso. A) Sitio Vuelta Coyoles, B) Sitio Poncho Mora y C) Sitio Trinidad

está a 8,5 m bajo la superficie del terreno. Los caudales captados varían entre 0,5 y 2 1/s, y captan lavas vacuolares y fracturadas.

El Sitio Trinidad (Figs. 2 y 3), se ubica en el cantón de Orotina, distrito La Ceiba, 13 km al oeste de la ciudad de Orotina, a una altitud entre 80 y 95 m s.n.m. En los alrededores de este sitio existen 
Cuadro 3

Características geológicas, hidrogeológicas y geotécnicas de los sitios seleccionados

\begin{tabular}{|c|c|c|c|c|c|}
\hline Nombre del sitio & Geología & $\begin{array}{l}\text { Nivel freático } \\
\text { (m) }\end{array}$ & $\begin{array}{l}\text { Espesor promedio } \\
\text { de suelo }(\mathrm{m})\end{array}$ & $\begin{array}{c}\text { Índice de plasticidad } \\
\text { del suelo }\end{array}$ & $\begin{array}{c}\text { Conductividad } \\
\text { hidráulica saturada } \\
(\mathrm{m} / \mathrm{s})\end{array}$ \\
\hline Vuelta Coyoles & $\begin{array}{l}\text { Avalancha } \\
\text { Ardiente }\end{array}$ & 11 & 3 & $\begin{array}{c}22.60 \% \\
\text { limo de alta } \\
\text { plasticidad }\end{array}$ & $\begin{array}{c}3,2 \times 10-7 \\
\text { Poco permeable }\end{array}$ \\
\hline Poncho Mora & $\begin{array}{l}\text { Avalancha } \\
\text { Ardiente }\end{array}$ & 25 & 1 & $\begin{array}{c}24.50 \% \\
\text { limo de alta } \\
\text { plasticidad }\end{array}$ & $\begin{array}{c}6,5 \times 10-7 \\
\text { Poco permeable }\end{array}$ \\
\hline Trinidad & $\begin{array}{l}\text { Formación } \\
\text { Tivives }\end{array}$ & 16 & 1.5 & $\begin{array}{c}29.50 \% \\
\text { limo de alta } \\
\text { plasticidad }\end{array}$ & $\begin{array}{c}3,4 \times 10-7 \\
\text { Poco permeable }\end{array}$ \\
\hline
\end{tabular}

13 pozos inscritos a menos de $1 \mathrm{~km}$ de distancia (Archivo Nacional de Pozos del SENARA), y los niveles freáticos varían entre 2 y $20 \mathrm{~m}$; sólo en 3 pozos el nivel está a menos de $3 \mathrm{~m}$ (Cuadro 6), por lo que la profundidad promedio del agua en el sitio es de $16 \mathrm{~m}$. Los caudales producidos varían entre 0,63 y 3,5 1/s, y captan aguas de almacenadas en tobas e ignimbritas principalmente. El espesor promedio de suelo arcilloso en el sitio es de 1,5 m (Cuadro 3). La geología en superficie corresponde con los depósitos de mesobrecha volcánica de la Formación Tivives (Denyer et al., 2003a)

\section{Cuadro 4}

Información de los pozos cercanos al Sitio Vuelta Coyoles

\begin{tabular}{|c|c|c|c|c|c|c|c|c|}
\hline Pozo & $\begin{array}{l}\text { Coordenadas } \\
\text { Este/Norte }\end{array}$ & Prof. (m) & $\mathrm{NE}(\mathrm{m})$ & $\mathrm{ND}(\mathrm{m})$ & Caudal (1/s) & Uso & Rejilla & $\begin{array}{c}\text { Litología / zona } \\
\text { captada }\end{array}$ \\
\hline RG-578 & $495525 / 215025$ & 50,00 & 8,00 & 18,00 & 2,00 & Doméstico & $16-46 \mathrm{~m}$ & $\begin{array}{c}\text { 16-22 lava } \\
\text { 22-32 toba } \\
\text { 32-42 ignimbrita }\end{array}$ \\
\hline RG-94 & $495800 / 213200$ & 45,00 & 10,70 & 0,50 & 0,50 & Doméstico & -- & -- \\
\hline RG-95 & $495400 / 213300$ & 45,60 & 9,12 & 0,50 & 0,50 & Doméstico & -- & -- \\
\hline RG-194 & $498600 / 213400$ & 43,00 & 6,00 & -- & 4,40 & Doméstico & $12-49 m$ & $\begin{array}{c}\text { 12-22 brecha } \\
\text { lávica } \\
\text { 22-49 lava }\end{array}$ \\
\hline RG-285 & $498250 / 213350$ & 39,00 & 13,50 & -- & 4,7 & Doméstico & $82-100 \mathrm{~m}$ & lava \\
\hline RG-263 & $495650 / 214300$ & 30,00 & 8,00 & -- & 0,50 & Doméstico & $9-30 \mathrm{~m}$ & $\begin{array}{l}\text { 9-22 coluvio } \\
22-30 \text { lava }\end{array}$ \\
\hline RG-269 & $498100 / 214650$ & 32,00 & 4,00 & 18 & 1,00 & Doméstico & $20-32 m$ & lavas \\
\hline RG-333 & $497050 / 215550$ & 83,00 & 14,00 & -- & 3,00 & Industrial & -- & $\begin{array}{c}\text { 16-26 ignimbrita } \\
26-46 \text { toba }\end{array}$ \\
\hline
\end{tabular}

Prof.: profundidad del pozo, NE: nivel estático, ND: nivel dinámico, -- Sin información Fuente: Archivo de Pozos del SENARA 
Información de los pozos cercanos al Sitio Poncho Mora

\begin{tabular}{|c|c|c|c|c|c|c|c|c|}
\hline Pozo & $\begin{array}{l}\text { Coordenadas } \\
\text { Este/Norte }\end{array}$ & $\begin{array}{l}\text { Prof. } \\
(\mathrm{m})\end{array}$ & $\mathrm{NE}(\mathrm{m})$ & $\mathrm{ND}(\mathrm{m})$ & $\begin{array}{c}\text { Caudal } \\
(1 / \mathrm{s})\end{array}$ & Uso & Rejilla & $\begin{array}{c}\text { Litología / zona } \\
\text { captada }\end{array}$ \\
\hline RG-166 & $491100 / 215100$ & 50,00 & 27,71 & -- & 0,82 & Doméstico & $49-50 \mathrm{~m}$ & lavas \\
\hline RG-229 & $488500 / 213200$ & 39,00 & 39,5 & -- & 2,00 & Doméstico & $40-50 \mathrm{~m}$ & lavas \\
\hline RG-683 & $489150 / 210400$ & 146,00 & 110,00 & -- & 0,50 & Riego & $128-146 m$ & lavas \\
\hline RG-692 & $492250 / 215270$ & 58,00 & 8,50 & 12,05 & 1,60 & Doméstico & $18-53 \mathrm{~m}$ & $\begin{array}{l}\text { 18-34 lava } \\
\text { vacuolar } \\
34-58 \text { lava } \\
\text { fracturada }\end{array}$ \\
\hline
\end{tabular}

Prof.: profundidad del pozo, NE: nivel estático, ND: nivel dinámico, -- Sin información

Fuente: Archivo de Pozos del SENARA

\section{DISCUSIÓN Y CONCLUSIONES}

Con base en los lineamientos definidos a partir de estudios anteriores y legislaciones existentes, y tomados como guía para determinar la aptitud de sitios para la disposición de desechos sólidos al oeste del Valle Central, se puede afirmar que las tres áreas propuestas, preliminarmente, cumplen con las condiciones para el desarrollo de grandes rellenos sanitarios; sin embargo es necesario realizar estudios de detalle en cada una.

A pesar de que los tres sitios presentan condiciones físicas de terreno similares, existen otros aspectos legales, económicos y paisajísticos que pueden ayudar a discriminar entre ellos.

Así por ejemplo, el sitio Poncho Mora, pese a ser amplio, presenta ciertos inconvenientes de índole legal, debido a que es el que presenta más servidumbres, cuatro simultáneas: una por la línea de transmisión eléctrica de alta tensión, otra por la tubería del oleoducto de la Refinadora Costarricense de Petróleo (RECOPE), otra por el derecho de vía de la autopista San José-Caldera y la última por la tubería del acueducto a Puntarenas. Dichas servidumbres le restan espacio e incomodarían durante una futura fase de operación.

El sitio Trinidad, en Orotina, por encontrarse en una región de relieve muy plano, presenta un mayor grado de exposición a los vientos y al entorno visual, que los otros dos sitios. Además, de las tres opciones, es la que se encuentra más alejada de San José, lo que incrementaría notablemente los costos.
Por el contrario, el sitio Vuelta Coyoles, en Atenas, no posee problemas de servidumbres ni de excesiva exposición visual, su área es mayor a la del sitio Poncho Mora y se encuentra ubicado más cerca de San José. Por lo tanto, se concluye que de los tres sitios propuestos, éste sitio es el más apto por sus condiciones físicas y su factibilidad.

\section{RECOMENDACIONES}

Para desarrollar adecuadamente un proyecto regional de relleno sanitario, que solvente toda la problemática de los desechos sólidos del Valle Central, recomendamos que futuros estudios exhaustivos se enfoquen en alguno de los sitios propuestos, con el fin de determinar definitivamente su idoneidad para el desarrollo de este tipo de proyectos.

La presente investigación no llevó a cabo estudios hidrogeológicos, geotécnicos, biológicos, forestales ni arqueológicos de detalle en los sitios propuestos, por lo que, antes de seleccionar definitivamente un sitio, se recomienda que se efectúen todos los estudios técnicos, físicos y socioeconómicos que especifique el Ministerio de Salud, la Comisión Nacional de Prevención de Riesgos y Atención de Emergencias (CNE) y en especial, la Secretaría Técnica Nacional Ambiental (SETENA), en sus respectivos términos de referencia.

Entre los estudios técnicos que se recomienda llevar a cabo, hacemos especial énfasis en los 


\section{Cuadro 6}

Información de los pozos cercanos al Sitio Trinidad

\begin{tabular}{|c|c|c|c|c|c|c|c|c|}
\hline Pozo & $\begin{array}{l}\text { Coordenadas } \\
\text { Este/Norte }\end{array}$ & Prof. (m) & $\mathrm{NE}(\mathrm{m})$ & $\mathrm{ND}(\mathrm{m})$ & Caudal (1/s) & Uso & Rejilla & $\begin{array}{l}\text { Litología / zona } \\
\text { captada }\end{array}$ \\
\hline BC112 & $466220 / 208850$ & 40,00 & 25,00 & 30,00 & 0,63 & Industrial & $25-40 m$ & toba lítica \\
\hline BC274 & $465630 / 208593$ & 60,00 & 15,00 & 25,00 & 1,05 & Riego & $\begin{array}{l}55-60 \mathrm{~m} \\
17-30 \mathrm{~m} \\
33-47 \mathrm{~m}\end{array}$ & $\begin{array}{l}\text { 17-24 ignimbrita } \\
24-60 \text { toba }\end{array}$ \\
\hline ВC396 & $466300 / 207900$ & 60,00 & 7,00 & 8,00 & 2,50 & Doméstico & $13-60 \mathrm{~m}$ & $\begin{array}{l}\text { 13-39 aluvión } \\
\text { 39-60 toba }\end{array}$ \\
\hline BC419 & $465450 / 208400$ & 70,00 & 30,00 & -- & 2,50 & Doméstico & $30-70 \mathrm{~m}$ & $\begin{array}{c}\text { 30-52 lava } \\
52-70 \text { arenisca }\end{array}$ \\
\hline BC426 & $466625 / 208100$ & 75,00 & 15,00 & -- & 3,00 & Industrial & $15-75 \mathrm{~m}$ & toba \\
\hline BC439 & $464925 / 209150$ & 64,00 & 25,00 & -- & 2,20 & Doméstico & $34-64 m$ & $\begin{array}{c}\text { 34-35 toba } \\
\text { 35-64 paleoaluvión }\end{array}$ \\
\hline ВC509 & $465500 / 208785$ & 90,00 & 19,85 & -- & 2,12 & Industrial & $\begin{array}{l}36-57 \mathrm{~m} \\
64-87 \mathrm{~m}\end{array}$ & $\begin{array}{l}\text { 36-64 toba cin- } \\
\text { erítica } \\
\text { 64-87 ignimbrita }\end{array}$ \\
\hline BC519 & $465750 / 208650$ & 103,00 & 3,00 & -- & 2,84 & Industrial & $\begin{array}{c}83-86 \mathrm{~m} \\
98-101 \mathrm{~m}\end{array}$ & $\begin{array}{l}83-85 \text { ignimbrita } \\
85-101 \text { toba }\end{array}$ \\
\hline BC574 & $465260 / 209050$ & 76,00 & 2,00 & -- & 2,4 & Público & $18-76 \mathrm{~m}$ & $\begin{array}{l}\text { 18-32 toba lítica } \\
\text { 32-58 andesita } \\
\text { 58-76 toba }\end{array}$ \\
\hline BC581 & $465650 / 208400$ & 100,00 & 22,57 & 32,37 & 1,50 & Industrial & $\begin{array}{l}57-63 \mathrm{~m} \\
69-72 \mathrm{~m}\end{array}$ & $\begin{array}{l}\text { 57-63 lava } \\
\text { 63-69 limo con } \\
\text { materia orgánica }\end{array}$ \\
\hline ВC600 & $466251 / 208338$ & 55,00 & 6,00 & 28,00 & 1,00 & $\begin{array}{l}\text { Doméstico- } \\
\text { Riego }\end{array}$ & $3-55 \mathrm{~m}$ & $\begin{array}{c}\text { 3-6 arcilla } \\
\text { 6-55 aluvión }\end{array}$ \\
\hline BC610 & $465920 / 208500$ & 68,00 & 25,00 & -- & 3,5 & Doméstico & $25-68 \mathrm{~m}$ & $\begin{array}{l}\text { 25-32 lavas al- } \\
\text { teradas (Aguacate) } \\
\text { 32-68 lava negra } \\
\text { 32-68 lava negra }\end{array}$ \\
\hline BC635 & $466379 / 208371$ & 64,00 & 10,00 & 40,00 & 1,5 & $\begin{array}{l}\text { Doméstico- } \\
\text { Riego }\end{array}$ & $40-64 m$ & toba lítica \\
\hline
\end{tabular}

Prof.: profundidad del pozo, NE: nivel estático, ND: nivel dinámico, -- Sin información

Fuente: Archivo de Pozos del SENARA

estudios hidrogeológicos, para determinar in situ el espesor de la zona no saturada, porosidades de las distintas capas, modelado del sistema de flujo, dirección de flujo del agua subterránea, gradiente hidráulico, tiempos de tránsito de contaminantes, entre otros.

Dada la problemática y poca voluntad demostrada por los municipios para manejar el asunto de los desechos, es recomendable que la ejecución y operación la realicen empresas particulares y que sea vigilada o controlada por el municipio y comunidad anfitriona.

El manejo y disposición final de desechos sólidos es un servicio cuyo costo todos debemos pagar. Por ello, esta actividad es lo suficientemente rentable para permitir que en el sitio se apliquen técnicas de ingeniería apropiadas para el desarrollo de un proyecto ejemplar, con el mínimo de impactos al ambiente. Además, dicho servicio permite la generación de ingresos económicos para 
el municipio o distrito anfitrión. Debido a esto, es importante que la comunidad se involucre con el proyecto, brindando vigilancia y seguimiento.

Como medida preventiva para la evitar la contaminación del agua subterránea, el Reglamento Nacional de Manejo, Disposición y Operación de los Desechos Sólidos de Costa Rica el reglamento vigente de Rellenos Sanitarios (La Gaceta N ${ }^{\circ} 206$, 23 octubre 1998: Decreto Ejecutivo No 27378-S) estipula un espesor de no menos de $50 \mathrm{~cm}$ de arcillas o de un adecuado material impermeabilizante, como constituyente principal del sello inferior del sitio de disposición final de desechos sólidos.

$\mathrm{Si}$ se decide transportar los desechos por vía férrea, se debe implementar una estación de transferencia, la cual ocuparía no menos de una hectárea. Por facilidad y ubicación, esta estación debería ubicarse en el borde oeste de la ciudad capital, siendo una posibilidad la zona de La Carpio, donde ya opera un relleno sanitario, o el oeste de Pavas, donde se ubican unos terrenos que funcionaron como canteras de extracción de materiales de construcción.

Es recomendable efectuar una campaña de información y divulgación en los municipios y comunidades del Valle Central, para que se conozcan los alcances, virtudes y ventajas de usar sitios apropiados para el desarrollo de rellenos sanitarios regionales.

\section{AGRADECIMIENTOS}

Este trabajo es resultado del Proyecto de Acción Social ED-1696 de la Vicerrectoría de Acción Social de la Universidad de Costa Rica. El Geólogo Claudio González brindó un apoyo invaluable en la recopilación y síntesis bibliográfica. El M.Sc. Rolando Mora y el personal técnico del Laboratorio de Geotecnia de la Escuela Centroamericana de Geología, Javier Alvarado, Luis Meneses y Maximiliano Garnier (asistente), colaboraron en las giras de campo, recolección, análisis, procesamiento e interpretación de muestras. El M.Sc. Gunther Schosinsky brindó asesoría hidrogeológica.

\section{REFERENCIAS}

ARIAS, O. \& DENYER, P., 1990: Geología de la Hoja Río Grande.- Escala 1:50 000, IGN, San José.

ASTORGA, A. \& AVILÉS, L., 1995: Estudio técnico de evaluación de sitios aptos para el desarrollo de rellenos sanitarios regionales en el Gran Área Metropolitana de San José.- 26 págs. Universidad de Costa Rica, San José [Informe interno].

DENYER,P.,AGUILAR,T.\&ALVARADO,G.E., 2003a: Geología y estratigrafía de la Hoja Barranca, Costa Rica.- Revista Geológica de América Central, 29: 105-125.

DENYER, P., MONTERO, W.\& ALVARADO, G.E., 2003b: Atlas tectónico de Costa Rica.- 79 págs. Ed. de la Universidad de Costa Rica, San José.

GTZ, 1991: Plan Nacional de Manejo de desechos de Costa Rica.- 193 págs. Agencia Alemana de Cooperación Técnica (GTZ ) - Gobierno de Costa Rica, San José [Informe interno].

KNOBLICH, K. \& MANZ. E., 1993: Discusión sobre los sitios para los vertederos de desechos sólidos de San José (Costa Rica).- Revista Geológica de América Central, 16: 95-101.

LABORATORIO DE SISTEMAS DE INFORMACIÓN GEOGRÁFICA EIF ITCR, 2000: Atlas Costa Rica 2000.- Instituto Tecnológico de Costa Rica [CD- ROM].

MONTERO, W., 1999: El terremoto del 4 de marzo de 1924 (Ms 7,0): ¿Un gran temblor interplaca relacionado al límite incipiente entre la Placa Caribe y la Microplaca Panamá?.- Revista Geológica de América Central, 22: 25-62.

SENARA, Base Nacional de Pozos. 
STEFFEN, H., 1995: Informe preliminar sobre la situación de sitios para rellenos sanitarios en el cantón de Mora.- 47 págs. Agencia Alemana de Cooperación Técnica (GTZ ), Alemania [Informe público SETENA, expediente $\mathrm{N}^{\circ}$ 579-04].

VAN WESTEN, C.J., 1997: Tools for map analysis applied to the selection of a waste disposal site.- En: ITC: ILWIS 2.1 for Windows, Applications Guide.- 341 págs. International Institute for Aerospace Survey and Earth Sciences, Enschede.

\section{Notas hemerográficas:}

La Gaceta N 206, 23 octubre 1998: Decreto 27378-S

La Gaceta No 50, 10 marzo 2006: Reglamento municipal sobre el manejo de residuos y rellenos sanitarios.

La Gaceta $\mathrm{N}^{\circ}$ 85, 4 mayo 2006: Decreto 32967-MINAE, Anexo 3. 